\title{
INFECTIONS BY MULTIDRUG RESISTANT ORGANISMS IN A NEUROSURGICAL AND NEUROPSYCHIATRIC CARE CENTRE AND THE CHANGING ANTIMICROBIAL SUSCEPTIBILITY PATTERNS.
}

\author{
R. Ravikumar, Jyothi E. K, Bornali Sarmah Dutta, Vidyasagar K, Nandan T. M, Ashalatha G
}

1. Professor and Head, Department of Neuromicrobiology, NIMHANS, Bangalore.

2. PhD Scholar, Department of Neuromicrobiology, NIMHANS, Bangalore.

3. Non-PG Senior Resident, Department of Neuromicrobiology, NIMHANS, Bangalore.

4. Non-PG Senior Resident, Department of Neuromicrobiology, NIMHANS, Bangalore.

5. Non-PG Senior Resident, Department of Neuromicrobiology, NIMHANS, Bangalore.

6. Post Doctoral Fellow in Neuroinfections, Department of Neuromicrobiology, NIMHANS, Bangalore.

\section{CORRESPONDING AUTHOR}

Dr. R. Ravikumar, Professor and Head, Department of Neuromicrobiology, NIMHANS, Bangalore-560029, E-mail: ravikumarbly@yahoo.co.uk, Ph: +919448073965

ABSTRACT: BACKGROUND: Infections contribute to major morbidity and mortality in the developing countries. Antimicrobial resistance is another major problem with the increasing infections and non availability of ideal antimicrobial agent. Despite the advances in antimicrobial therapy, infections remain a serious burden. This study was designed to assess the increasing infection rates and antimicrobial susceptibility patterns in neurosurgical and neuropsychiatric patients.

Over a period of three years and six months from January 2009 to June 2012, various samples were processed, isolated the infectious agent and identified. The different samples included samples like CSF, urine, pus, tracheal aspirates, shunt \& catheter tips, tissue and blood. These were assessed for antimicrobial susceptibility patterns by conventional and automated culture and sensitivity systems. The results were then statistically analyzed.

This study found that in general, there is a trend towards increased rates of infections, the majority of which were caused by Multi Drug Resistant Gram Negative Bacilli (MDRGNB), followed by Candida species and Cryptococcus species, Methicillin Resistant Staphylococcus aureus (MRSA), and Mycobacterium tuberculosis. The infection rates, from January 2009 to June 2012, were $3.7 \%$ to $5.1 \%$ for MDRGNB, $2.89 \%$ to $4.24 \%$ for Candida, $2.93 \%$ to $3.13 \%$ for Cryptococcus, $2.8 \%$ to $2.1 \%$ (till June 2012) for MRSA and $1.3 \%$ to $5.7 \%$ for Mycobacterium tuberculosis. Also, a trend towards increasing resistance among the isolates to the carbapenem antibiotics was observed.

The increasing trend of infections is a cause for concern throughout the country. Indiscriminate use of antibiotics, hospital personnel not adhering to safety precautions etc are the main reasons for the increased infection rates. Newer therapeutic agents and strict antibiotic policies are required to effectively control the issue of emerging infections.

KEY WORDS: MDRGNB, MRSA, infections, carbapenems, resistance.

INTRODUCTION: The burden of health care associated infection in developing countries is high. Despite stringent precautions and policies, the infection rates are still rising and the control of infections remains an elusive goal. The prevalence of infections varies widely from region to 
region in our country. In our country we are especially vulnerable to infections caused by resistant strains due to the overwhelmingly indiscriminate use and across the counter availability of antibiotics ${ }^{1}$. Development of carbapenem and multi drug resistant Gram negative bacterial strains have led to infections that are hard to treat and add to the physical, mental and financial burden for the patient and the community. A systematic, regional periodic synopsis of the prevalence of the various infections would not only help to study the changing trends of infections, but also to assess the effectiveness of the various control programs. The clinician needs to be aware about the patterns of infections and resistance and of the epidemiological observations in the various wards of the hospital, along with the observations and interpretations from the microbiology laboratory. Intercommunication and cooperation among the microbiologists and clinicians is absolutely essential for the effective treatment of infections.

The immense importance of microbiologic surveillance has been brought into focus by the emergence of newer pathogenic strains as well as multi drug resistant strains ${ }^{2}$. The etiological agents as well as the susceptibility patterns keep changing with time and control policies and it is essential that the data about these be refreshed so as to aid in the development of effective antibiotic policies and hospital infection surveillance programs. This will also help in putting an end to indiscriminate antibiotic prescription and thereby reduce emergence of resistant microorganisms.

The current study was conducted to assess the changing trends in the rate of various infections and the changing patterns of antimicrobial susceptibility in neurosurgical and neuropsychiatric care centre, over the last three and a half year period. In our centre, we commonly encounter neurological cases of chronic \& acute pyogenic meningitis, immunocompromised cases with various neuroinfections, various neurodegenerative conditions etc; neurosurgical cases of trauma, shunt infections, post operative infections, hydrocephalus, malignancies etc; and neuropsychiatric conditions which include various psychological syndromes, infectious cases like neurosyphilis, substance abuse related infections etc. Thus, it provides an excellent set up to study various types of infections and their etiologies.

MATERIALS AND METHODS: The study was conducted over a period of three and a half years, from January 2009 to June 2012. All samples that came into the microbiology laboratory during this period were evaluated.

SAMPLES PROCESSED DURING THE STUDY: CSF, blood, tracheal aspirates, urine, tissue, pus, swabs, shunt \& catheter tips etc.

COLLECTION: The samples were collected from the patients at the respective hospital wards and transported immediately in a sterile container to the microbiology laboratory.

PROCESSING OF THE SAMPLES:CSF specimens were subjected to gross examination and cell count. Urine and tracheal specimens were stained using Gram's staining technique to identify the Gram's character and morphology of bacteria. The routine samples included, tracheal aspirates, tissue, pus, swabs, shunt \& catheter tips etc. These were cultured on Thioglycollate broth, Blood agar and Mac Conkey's agar and incubated overnight at $37^{\circ} \mathrm{C}$. Urine samples were cultured on cystine lactose electrolyte deficient medium (CLED) and blood agar and incubated as for the other samples ${ }^{3}$. Blood was cultured conventionally and also with automated BacT/ ALERT system. 
After the overnight incubation, the culture plates were read and Gram's staining was done so as to derive the Gram's nature of the isolates. Based on the biochemical characteristics, the isolates were identified conventionally. Also, antibiotic sensitivity testing was carried out by conventional Kirby- Bauer disc diffusion method as per the CLSI guidelines 4 .

All Gram positive cocci in clusters were tested for coagulase production. The Gram negative bacilli were tested for indole production, mannitol fermentation, motility and also tested on triple sugar iron agar medium for obtaining the fermentation pattern ${ }^{3}$.

0.5Mc Farland's standards of inoculum were plated on to Meuller Hinton agar (MHA) to make lawn cultures on which Kirby Bauer disc diffusion for antibiotic sensitivity testing was carried out ${ }^{4}$.

Vitek 2 compact 60 automated culture and sensitivity system was used to confirm the identification and antibiogram of the isolates ${ }^{5,6}$.

For the isolation of Mycobacterium, CSF was cultured on Lowenstein Jensen's (LJ) medium $^{3}$ and also by Mycobacterium Growth Indicator Tube (MGIT) automated technique ${ }^{7}$. The culture was read each week for 8 weeks before pronouncing as 'no- growth'. The antimycobacterial drug susceptibility was also tested using the MGIT system. Strains were genotyped to look for the presence mutant bands coding for Isoniazid and Rifampicin resistance, using Genotype MTBDRplus 96 assay.

India ink staining was done for the cryptococci for the demonstration of the capsule. Latex agglutination test (Cryptococcal antigen test- CRAg) for the identification of the capsular polysaccharide antigen was done for all doubtful cases. The samples were seeded onto the Sabouraud's Dextrose agar (SDA) medium and incubated at room temperature ${ }^{8}$. Incubation was continued for four weeks before declaring it as 'no growth'. Species level identification was based on the biochemical reactions which included caffeic acid test and urease test.

STATISTICAL ANALYSIS: The results were tabulated and the data was analysed using simple statistical tools like measures of central tendency like mean, and percentage calculations etc.

RESULTS: Over the three years and six months study, 35,543 samples were cultured on liquid and solid media for the isolation of the etiological agent. The year and sample wise distribution is given in Table 1. The rates of mycobacterial and cryptococcal isolation from CSF samples were as given in Table 2.

The study revealed that there is a trend, in general, towards an increased rate of infections during the study period. The results have been consolidated in the figures 1- 4 based on the samples from which the organisms were isolated.

Out of all the isolates, multi drug resistant Gram negative bacilli (MDRGNB) topped the list with the most number of isolates. In 2009 from routine samples it was 3.65\% of all the samples processed. In the subsequent years, from 2010 to June 2012, the incidence rates were $1.12 \%, 3.81 \%, 5.08 \%$ respectively. Among the blood and CSF samples MDRGNB were found to be less in number, as expected considering their sterile nature. From blood, no MDRGNB were isolated during 2009. From 2010 to 2011 it was $0.69 \%$ and $0.14 \%$ respectively. Till the end of the month June 2012, no Gram negative isolates from blood samples were found to be multi drug resistant. CSF sample isolates were respectively $0.08 \%, 0.27 \%, 0.52 \%$ and $1.35 \%$ from 2009 to 2012.

MDRGNB were followed by Candida species, isolated mostly from urine samples. In 2009 , it was $2.89 \%$ from routine samples, while CSF samples and blood samples had $0.05 \%$ and $0.19 \%$ respectively. There was a slight dip in the percentage isolation $(2.67 \%)$ from routine 
samples in 2010 when compared to the previous year. But steady increase was found in the subsequent years with $3.24 \%$ and $4.24 \%$ respectively, during 2011 and 2012 . Blood isolates of Candida increased to $0.21 \%$ in 2010 but fell to $0.14 \%$ in 2011 , and again increased to $0.25 \%$ in 2012 (upto June). CSF isolates were $0.23 \%$ in 2010, 0.34\% in 2011 and $0.06 \%$ in 2012. Refer Fig 1 and 2.

CSF Cryptococcal isolates also showed an incidence of $2.93 \%$ in $2009,2.76 \%$ in 2010 , 3.73\% in 2011 and 3.13\% in 2012. Refer Fig 3.

MRSA had $2.77 \%$ isolation from routine samples during the first year of study. The subsequent years showed an incidence rate of $2 \%, 3.79 \%$ and $2.12 \%$ respectively during 2010 , 2011 and 2012. From blood samples, the MRSA isolation rates were $0.39 \%, 0.35 \%, 1.44 \%$ and $0.25 \%$ from 2009 to 2012. From CSF samples, the rates were, from 2009 to $2012,0.16 \%, 1.18 \%$, $1.07 \%$, and $1.03 \%$. Refer Fig 3.

Mycobacterium tuberculosis isolates from CSF samples showed an incidence rate of $1.26 \%, 0.94 \%, 3.36 \%$, and $5.67 \%$ over the three and a half year study period, ie from 2009 to 2012.

Most of the isolates from routine samples were Gram negative in nature. CSF sample isolates, on the other hand showed more number of Gram positive isolates. The strains of Gram negative bacteria isolated during the study period was predominantly Escherichia coli, Non fermenting Gram negative bacilli(NFGNB), Pseudomonas species, Klebsiella species, Enterobacter species etc. Coagulase negative staphylococci (CONS) were the predominant isolate from CSF samples. The isolates of Enterobacteriaceae are closely followed by the staphylococcal isolates.

During the period of the study, a change in the pattern of the isolate picture was not observed except that most of the MDRGNB were of non-fermenting nature. Most of the NFGNB strains were identified by Vitek 2C60 to be Acinetobacter species.

Most of the isolates were found to be resistant to Ampicillin. In the first line antibiotics against the Gram negative bacilli, Amikacin and Gentamycin were found to be the most sensitive. Among the second line of antibiotics, Imipenem had around 98\% percent sensitivity. Piperacillin- Tazobactum combination was also found to have better efficacy towards the first line drug- resistant isolates. But, the study found that there is a shift towards increasing resistance to carbapenem antibiotics like Imipenem and Meropenem. More and more number of isolates was found to be Imipenem resistant as the study progressed. Further studies are required for statistical accounting of these data.

DISCUSSION: The rates of infections and the antimicrobial susceptibility patterns of different isolates depend on various factors. The chances of getting infections are high in individuals who are immunocompromised, in extremes of age, and in pregnancy ${ }^{9}$. Also, it depends on an individual's occupation, hygiene practises which inturn is heavily dependent on one's socioeconomic status, and public health awareness. Patients in a hospital are more prone to infections depending on their condition, place of stay (i.e. ICU, general wards etc), duration of stay etc ${ }^{10}$.

Owing to the indiscriminate use of antimicrobial agents, which include self medication, use of inappropriate drug, not completing the course of the drug etc, has contributed heavily towards the emergence of the newer multi drug resistant strains. Unrestricted use of antimicrobial agents in animals/ animal feeds, fish, and on plants over a relatively long period of 
time has resulted in an environment that has encouraged the development of resistance in many bacterial species ${ }^{11,12}$.

Longer lasting infections also contribute towards development of resistance. In the community, all these factors along with the microbes' natural tendency to evolve, are responsible for the increase in rates of infections with MRSA, Vancomycin resistant Enterococcus, Gram negative strains carrying extended spectrum beta lactamases (ESBL) \& metalobetalactamases etc.

With this retrospective study, we report our experience regarding the existing trends in incidence rates of infections with special reference to the rates of infections caused by MDRGNB and MRSA. Also, we report the rates of incidence and isolation of opportunistic fungal pathogens like Cryptococcus neoformans and Candida species. Cryptococcal strains were mainly isolated from immunocompromised individuals.

MRSA has become increasingly prevalent worldwide since it was first reported among hospitalized patients in a British hospital13, 14. MRSA has arisen as a major blood stream pathogen, as corroborated by the present study. Similar trend has been reported in the data from the West over the last two decades. MRSA is a major nosocomial pathogen and presents a serious issue in morbidity control among hospitalized patients ${ }^{15}$. MRSA strains are also highly prone to acquire resistance to other drugs to which these were earlier susceptible. In another unpublished study from our centre, it was found that none of the MRSA isolates were Linezolid resistant. But, it is important to have the knowledge of the existing susceptibility patterns and also, a strong surveillance system to screen for emerging and re-emerging patterns of resistance.

We also present in this study the corresponding data on Mycobacterium tuberculosis isolates from cerebrospinal fluid samples. The drastic increase from 3.13\% to 5.08\% isolation rate of the tubercle bacilli from the CSF samples can be attributed to the change in isolation method, from January 2012 onward. The isolation technique used, parallel to the conventional culture on LJ medium, was automated Mycobacterium Growth Indicator Tube system which employs Middlebrook's liquid medium for the isolation and also has better sensitivity than the conventional culture methods.

In the present study, most of the results obtained by manual/conventional methodologies were confirmed with automated culture and sensitivity systems. This would help in eliminating false positive results to a greater extend. One of the limitations of the present study is that, being a single centre study the results cannot be widely extrapolated. Also generalisation of surveillance data will be inaccurate as it depends on the institutional antibiotic policies, the type of patients and the community it is associated with. However, we believe that the data presented here would help in giving a greater picture about the need for strict antibiotic policies, hospital surveillance units, maintaining of universal safety precautions, efficient hygiene practises and spreading of awareness among the general public.

It is concluded that, there is increase in the rates of multi drug resistant isolates and therefore the infections caused by these organisms. Both Gram positive and Gram negative organisms are responsible for these infections and the opportunistic pathogens are responsible for increased morbidity among immunocompromised patients. Also, this study stresses on the requirement of strict antibiotic policies for hospital and other health care settings.

AKNOWLEDGMENT: The authors thank the support received from the Director/Vice Chancellor of National Institute of Mental Health and Neuro Sciences (NIMHANS), Bangalore 
and the assistance from the technical and supporting staff of the Department of Neuromicrobiology, NIMHANS.

Table 1: Year wise distribution of samples and etiological agents

\begin{tabular}{|c|c|c|c|c|c|c|c|c|}
\hline \multirow{4}{*}{ Isolates } & \multicolumn{6}{|c|}{ January to December } & \multicolumn{2}{|c|}{ January to June } \\
\hline & \multicolumn{2}{|c|}{2009} & \multicolumn{2}{|c|}{2010} & \multicolumn{2}{|c|}{2011} & \multicolumn{2}{|c|}{2012} \\
\hline & \multicolumn{8}{|c|}{ Isolate from routine samples } \\
\hline & No & $\%$ & No & $\%$ & No & $\%$ & No & $\%$ \\
\hline Candida & 161 & 2.89 & 150 & 2.67 & 187 & 3.24 & 126 & 4.24 \\
\hline MRSA & 154 & 2.77 & 112 & 2.0 & 219 & 3.79 & 63 & 2.12 \\
\hline MDRGNB & 203 & 3.65 & 63 & 1.12 & 220 & 3.81 & 151 & 5.08 \\
\hline $\begin{array}{c}\text { Total no of samples } \\
\text { processed }\end{array}$ & \multicolumn{2}{|c|}{5556} & \multicolumn{2}{|c|}{5604} & \multicolumn{2}{|c|}{5776} & \multicolumn{2}{|c|}{2970} \\
\hline Isolates & \multicolumn{8}{|c|}{ Isolate from blood samples } \\
\hline Candida & 4 & 0.19 & 3 & 0.21 & 2 & 0.14 & 1 & 0.25 \\
\hline MRSA & 8 & 0.39 & 5 & 0.35 & 20 & 1.44 & 1 & 0.25 \\
\hline MDRGNB & 0 & 0 & 1 & 0.69 & 2 & 0.14 & 0 & 0 \\
\hline $\begin{array}{c}\text { Total no of samples } \\
\text { processed }\end{array}$ & \multicolumn{2}{|c|}{2061} & \multicolumn{2}{|c|}{1445} & \multicolumn{2}{|c|}{1390} & \multicolumn{2}{|c|}{399} \\
\hline Isolates & \multicolumn{8}{|c|}{ Isolate from CSF samples } \\
\hline Candida & 2 & 0.05 & 5 & 0.23 & 10 & 0.34 & 1 & 0.06 \\
\hline MRSA & 6 & 0.16 & 26 & 1.18 & 31 & 1.07 & 16 & 1.03 \\
\hline MDRGNB & 3 & 0.08 & 6 & 0.27 & 15 & 0.52 & 21 & 1.35 \\
\hline $\begin{array}{c}\text { Total no of samples } \\
\text { processed }\end{array}$ & \multicolumn{2}{|c|}{3675} & \multicolumn{2}{|c|}{2200} & \multicolumn{2}{|c|}{2907} & \multicolumn{2}{|c|}{1560} \\
\hline
\end{tabular}

Table 2: Isolation pattern of Mycobacterium tuberculosis and Cryptococcus neoformans from CSF samples.

Journal of Evolution of Medical and Dental Sciences/Volume1/ Issue4/October - 2012 Page 449 
ORIGINAL ARTICLE

\begin{tabular}{|c|c|c|c|c|c|c|c|c|}
\hline \multirow{3}{*}{ Isolates } & \multicolumn{6}{|c|}{ January to December } & \multirow{2}{*}{\multicolumn{2}{|c|}{$\begin{array}{c}\text { January to June } \\
2012\end{array}$}} \\
\hline & \multicolumn{2}{|c|}{2009} & \multicolumn{2}{|c|}{2010} & \multicolumn{2}{|c|}{2011} & & \\
\hline & No & $\%$ & No & $\%$ & No & $\%$ & No & $\%$ \\
\hline $\begin{array}{l}\text { Mycobacterium } \\
\text { tuberculosis }\end{array}$ & 30 & 1.26 & 23 & 0.94 & 54 & 3.36 & 57 & 5.67 \\
\hline $\begin{array}{c}\text { Total no of samples } \\
\text { processed }\end{array}$ & \multicolumn{2}{|c|}{2378} & \multicolumn{2}{|c|}{2455} & \multicolumn{2}{|c|}{1607} & \multicolumn{2}{|c|}{1005} \\
\hline $\begin{array}{l}\text { Cryptococcus } \\
\text { neoformans }\end{array}$ & 80 & 2.93 & 82 & 2.76 & 80 & 3.73 & 42 & 3.13 \\
\hline $\begin{array}{c}\text { Total no of samples } \\
\text { processed }\end{array}$ & \multicolumn{2}{|c|}{2729} & \multicolumn{2}{|c|}{2968} & \multicolumn{2}{|c|}{2146} & \multicolumn{2}{|c|}{1342} \\
\hline
\end{tabular}

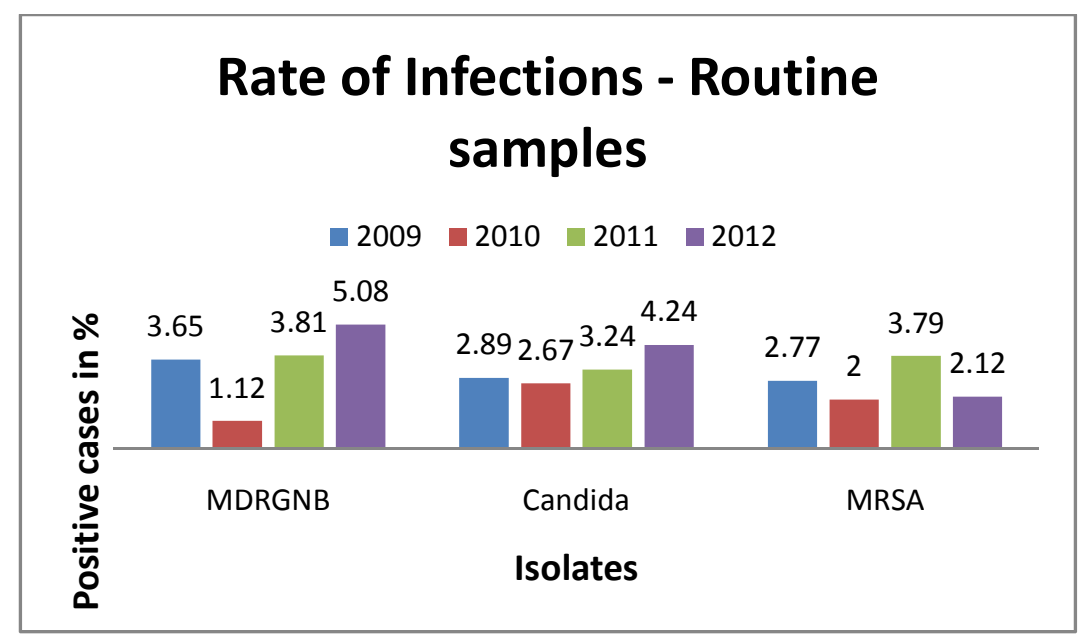

Fig1: Percentage of different isolates from the routine samples.

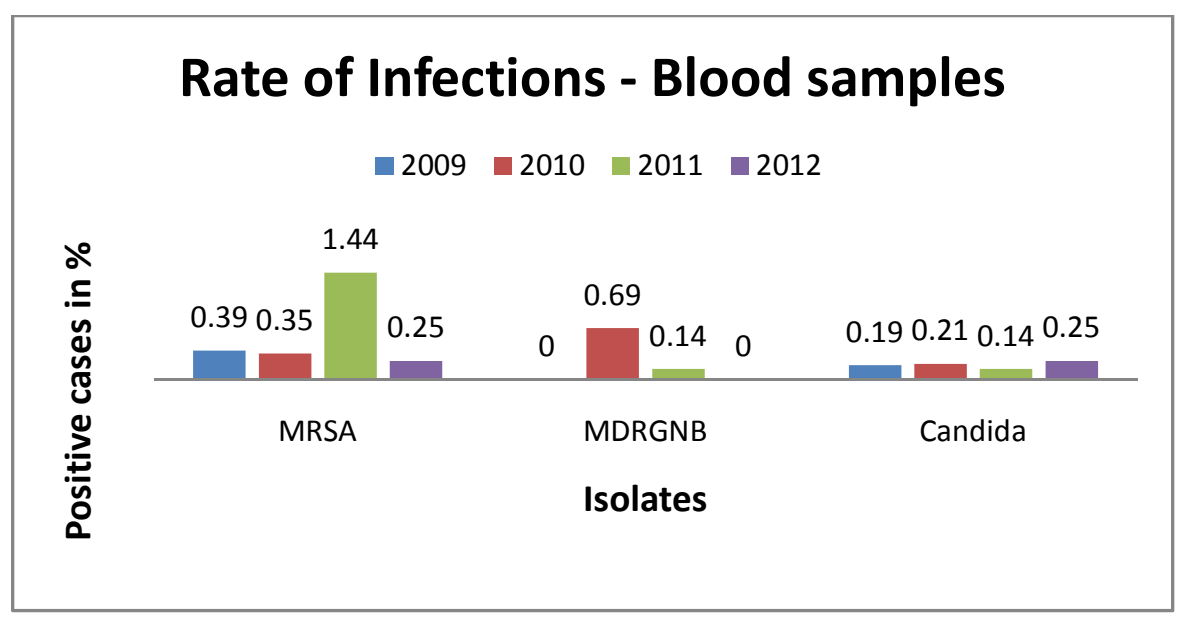

Fig 2: Percentage of different isolates from the blood samples.

Journal of Evolution of Medical and Dental Sciences/Volume1/ Issue4/October - 2012 Page 450 


\section{Rate of Infections - CSF samples}

$\square 2009-2010 \quad 2011 \square 2012$

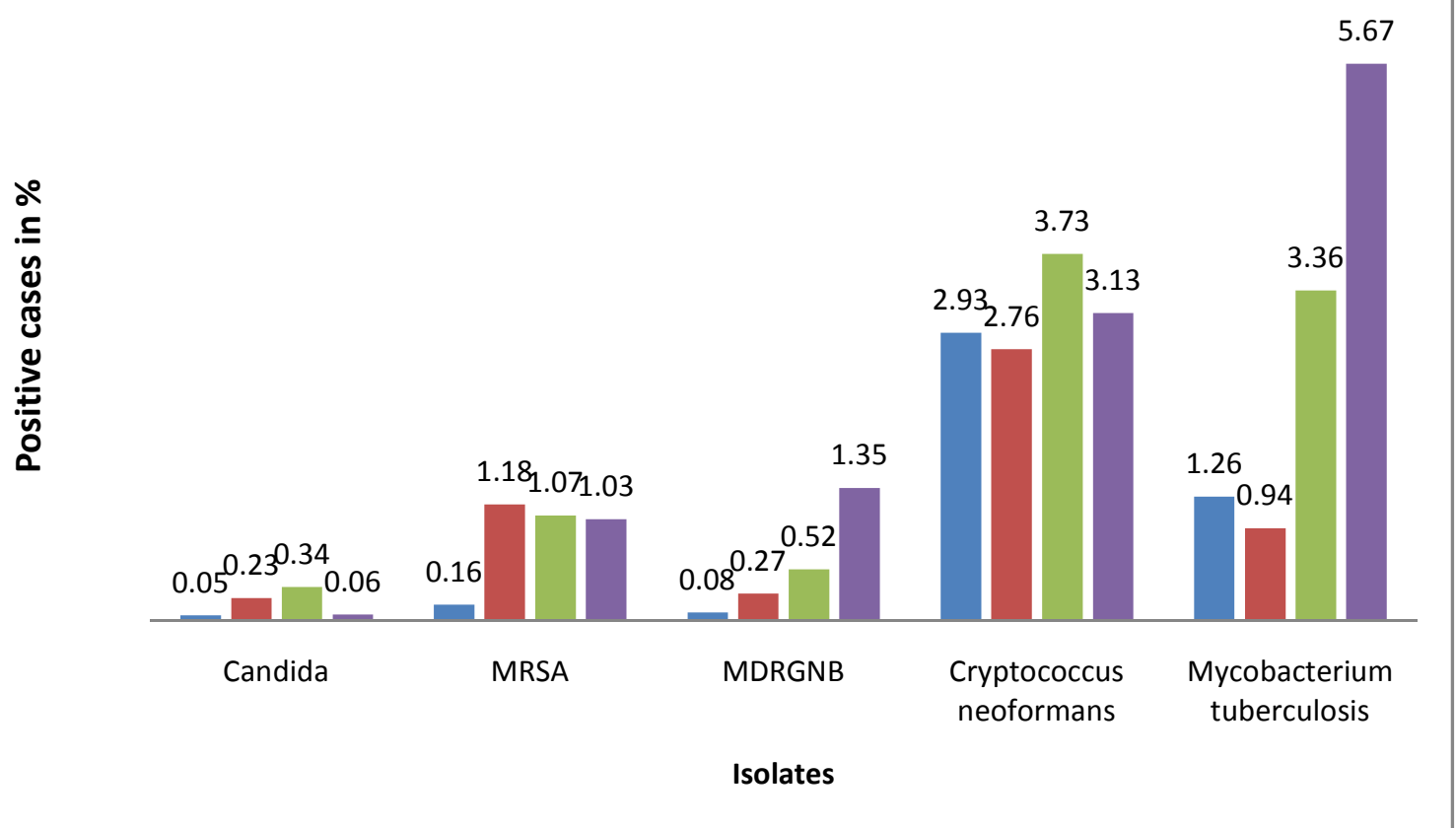

Fig3: Percentage of different isolates from Cerebrospinal fluid.

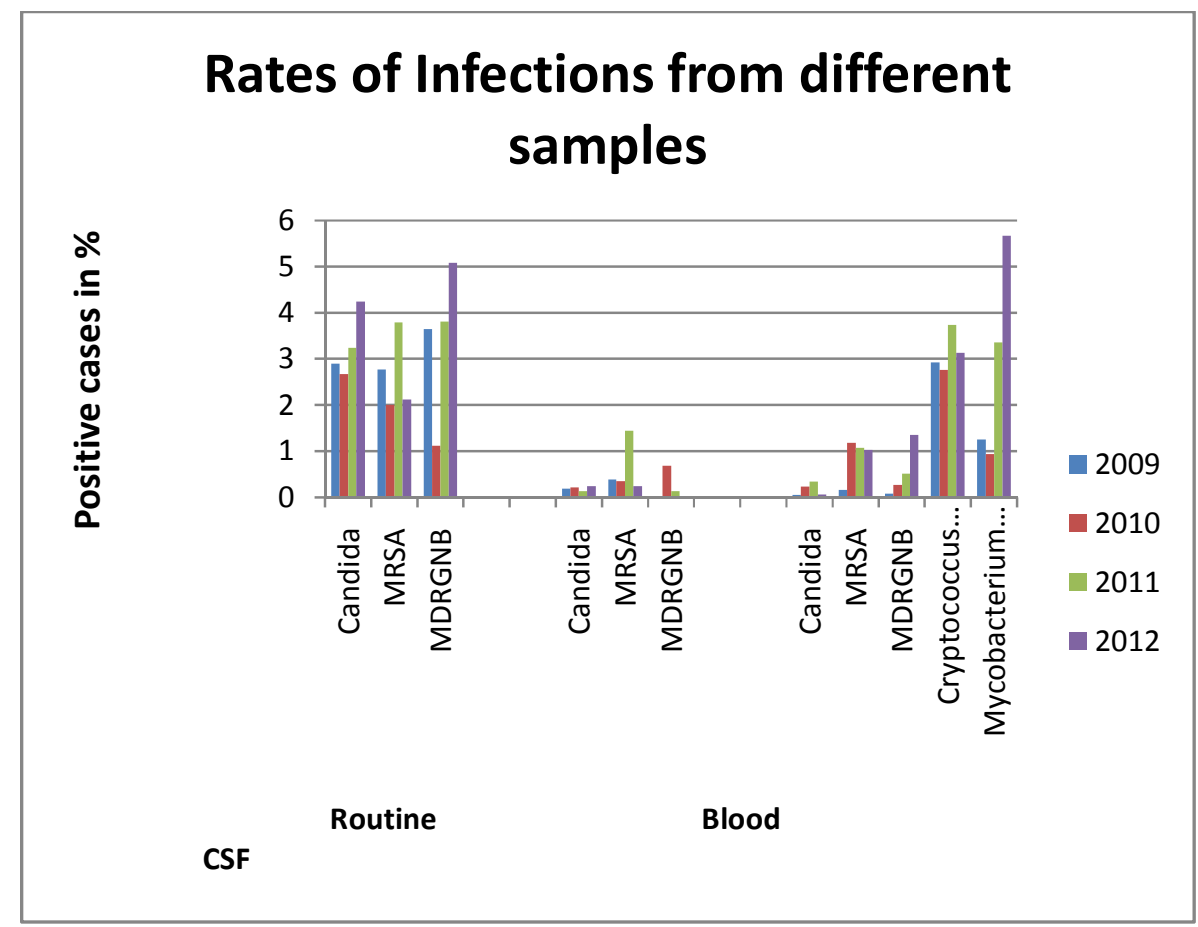

Fig 4: Percentage of different isolates from the various samples. 


\section{REFERENCES:}

1. Wattal C, Goel N, Oberoi JK, Raveendran R, Datta S,Prasad KJ. Surveillance of Multidrug Resistant Organisms in a Tertiary Care Hospital in Delhi, India. Supplement to JAPI.2010;58.32-36.

2. Raghunath D. Emerging antibiotic resistance in bacteria with special reference to India. J Biosci 2008; 33:593-603

3. Forbes BA, Sham DF, Weissfeld AS, editiors. Overview conventional methods for bacterial identification. Chapter B: Baliley and Scott's Diagnostic Microbiology, 10th ed. St.Louis: The CV Mosby Company; 1998.p167.

4. Clinical and Laboratory Standards Institute. Performance Standards for Antimicrobial Susceptibility Testing; Twenty-Second Informational Supplement. $9^{\text {th }}$ Ed. CLSI Document M100-S22. 2012;32(3). Wayne, PA.

5. Chatzigeorgiou K, Sergentanis TN, Tsiodras S, Hamodrakas SJ, Bagos PG. Phoenix 100 versus Vitek 2 in the Identification of Gram-Positive and GramNegative Bacteria: a Comprehensive Meta-Analysis. J Clin Microbiol. 2011;49(9):3284-91.

6. de Cueto M, Ceballos E, Martinez-Martinez L, Perea EJ, Pascual A. Use of Positive Blood Cultures for Direct Identification and Susceptibility Testing with the Vitek 2 System. J Clin Microbiol. 2004;42(8):3734-38.

7. 7.Bemer P, Palicova F, Rüsch-Gerdes S, Drugeon HB, Gaby E, Pfyffer GE. Multicenter Evaluation of Fully Automated BACTEC Mycobacteria Growth Indicator Tube 960 System for Susceptibility Testing of Mycobacterium tuberculosis. J Clin Microbiol. 2002;40(1):150-54.

8. Champa Saha D, Xess I, Biswas A, Bhowmik DM, Padma MV. Detection of Cryptococcus by conventional, serological and molecular methods. J Med Microbiol August 2009;58(8):1098-105.

9. Stefan H. Kaufmann E, Michael W. Steward.Topley and Wilson's Microbiology and Microbial Infections, Immunology, 10th Edition 2007.

10. Atul K Patel, Ketan K Patel, Kamlesh R Patel, Sanjiv Shah, Pratibha Dileep. Time trends in the epidemiology of microbial infections at a tertiary care center in West India over last 5 Years. Supplement to JAPI 2010;58:37-40.

11. Antimicrobial Resistance: An Ecological Perspective. Report from the American academy of microbiology; Based on an American Academy of Microbiology colloquium. July 16-18, 1999: San Juan, Puerto Rico.

12. Witte W. Impact of antibiotic use in animal feeding on resistance of bacterial pathogens. In Antibiotic Resistance: origins, evolution, selection and spread. John Wiley \& Sons, Ltd. Chichester, West Sussex, England. 1997

13. Barber M. Methicillin resistant Staphylococci. J Clin Path 1961;14:385-93.

14. Colley EW, Mc Nicol MW, Bracken PM. Methicillin-resistant staphylococci in a general hospital. Lancet 1965; 191:595-97.

15. Tenover FC, Gaynes RF. The epidemiology of Staphylococcus aureus. In Fischett VA, Novick RP, Ferretti JJ, Portnoy DA, Rood JI , editors. Gram-positive pathogens. Washington D C: American Society for Microbiology. 2000:414-21. 